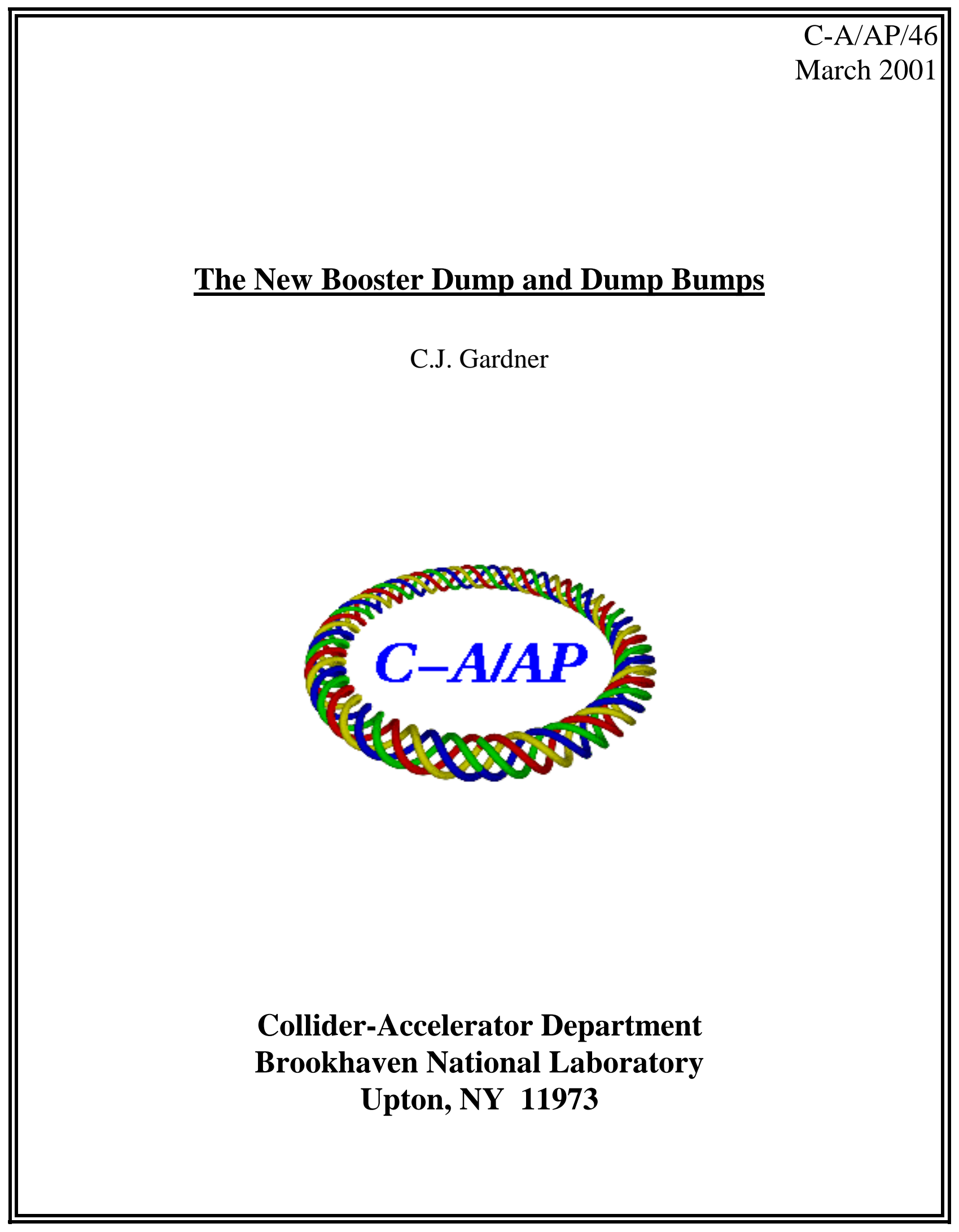




\title{
The New Booster Dump and Dump Bumps
}

\author{
C.J. Gardner
}

March 30, 2001

\section{Introduction}

One of the modifications [1] of the AGS Booster necessary for the Booster Applications Facility (BAF) currently under construction, is the installation of a septum magnet in the D6 straight section. This means that the D6 beam dump currently housed there will have to be removed. A new dump will then be installed in the B6 straight section which is currently empty.

The new dump is identical to the old one in design and dimension, and the plan is to place it initially in the B6 straight exactly as the old one is placed in the D6 straight. Unlike the stand that supports the D6 dump, the one that supports the B6 dump can be moved horizontally (radially) and vertically, allowing for adjustment of the horizontal and vertical apertures of the dump relative to the beam center line. After the initial placement, this feature will be utilized in studies to find the most effective positions for the apertures. The placement of the dumps is based on the apertures in Booster and calculations of the resulting acceptance envelope. These are revisited in this note along with calculations of the required orbit bumps in the dump region. Sections $2-4$ give a review of the horizontal and vertical bumps produced by the correction dipoles and the flat trim windings on the Booster dipoles. The Booster apertures and acceptance envelopes are reviewed in Section 5. Finally, in Section 6 the preferred positions for the B6 dump apertures are determined and the requirements for the horizontal and vertical dump bumps are given. 


\section{Booster Dipole Windings}

The Booster dipole has a main winding consisting of 16 turns, a flat trim winding consisting of 2 turns, and additional low-current trim windings consisting of 4 turns. The magnetic fields produced by the main and flat trim windings are respectively

$$
B=K I, \quad \Delta B=(K / 8) \Delta I
$$

where $I$ and $\Delta I$ are the corresponding currents and $K=2.43$ Gauss/A as reported by Thern [2]. For given values of $\Delta B / B$ and $I$, the current required in the flat trim winding is

$$
\Delta I=8 I(\Delta B / B) .
$$

Now, a proton with kinetic energy $W$ has momentum given by

$$
c p=\sqrt{W^{2}+2 m c^{2} W}
$$

and the magnetic rigidity in units of $\mathrm{Tm}$ is

$$
B \rho=\left(10^{6} / 299792458\right) p
$$

where $p$ is the momentum in units of $\mathrm{MeV} / c$. (The proton mass is

$m=938.271998 \mathrm{MeV} / c^{2}$ ). The field required in the Booster dipole is then

$$
B=B \rho / \rho
$$

where $\rho=13.8656$ meters is the nominal radius of curvature in the magnets. The fields and currents for protons with various kinetic energies are listed in Table 1 . Here $I$ is the current required in the main winding to give field $B$, and $\Delta I$ is the current required in the flat trim to give $\Delta B / B=0.01$. The maximum steady current allowed in the flat trim windings is $300 \mathrm{~A}$ (as per Roger Bonati).

\section{The D6 and B6 Horizontal Dump Bumps}

The AGS Booster has 6 superperiods with 4 FODO cells per superperiod. At a tune of 4.8 , the betatron phase advance per cell is $4.8 \times 360^{\circ} / 24=72^{\circ}$. The phase advance for 5 cells is then $360^{\circ}$. It follows that two dipole kicks placed 5 cells apart and excited with currents of equal 
Table 1: Booster Dipole Fields and Currents

\begin{tabular}{|c|c|c|c|c|}
\hline$W(\mathrm{MeV})$ & $B \rho(\mathrm{Tm})$ & $B$ (Gauss) & $I(\mathrm{~A})$ & $\Delta I(\mathrm{~A})$ \\
\hline 200 & 2.149636 & 1550 & 638 & 51.0 \\
400 & 3.183078 & 2296 & 945 & 75.6 \\
600 & 4.066099 & 2933 & 1207 & 96.6 \\
800 & 4.881030 & 3520 & 1449 & 116 \\
1000 & 5.657373 & 4080 & 1679 & 134 \\
1200 & 6.409165 & 4622 & 1902 & 152 \\
1400 & 7.144162 & 5152 & 2120 & 170 \\
1600 & 7.867073 & 5674 & 2335 & 187 \\
1800 & 8.580951 & 6189 & 2547 & 204 \\
2000 & 9.287881 & 6699 & 2757 & 221 \\
\hline
\end{tabular}

magnitude but opposite polarity, will produce a Lambda Bump with little or no COD (Closed Orbit Distortion) outside the Bump region. The D6 Dump Bump is the superposition of two such bumps. One lambda bump is produced by the flat trim windings on dipoles $\mathrm{C} 7$ and $\mathrm{E} 1$; the other by the windings on dipoles D2 and E4. The relative polarities of the dipoles are $-\mathrm{C} 7,+\mathrm{D} 2,+\mathrm{E} 1$, and $-\mathrm{E} 4$, where "+" and "-" indicate that the currents in the flat trim windings are respectively parallel and anti-parallel to the currents in the main windings. The B6 Dump Bump will follow the same scheme with the flat trim windings on dipoles A7, B2, C1, and C4. These will be connected together into one series string with relative polarities $-\mathrm{A} 7,+\mathrm{B} 2,+\mathrm{C} 1$, and $-\mathrm{C} 4$. Note that in this configuration there is no net EMF induced in the string from the main windings of the dipoles. The resistance and inductance of the series string for the D6 dump bump have been measured by Roger Bonati. He finds $0.105 \mathrm{Ohms}$ and $220 \mu \mathrm{H}$ respectively. The series string for the B 6 dump bump is expected to have the same values provided the length of cable in the string is the same.

Figures 1 and 2 show the B6 dump bump for the case in which the string of flat trims is excited with current $\Delta I$ such that $\Delta B / B=0.01$ in dipole B2. The closed orbit was determined by the MAD code with the horizontal and vertical tunes taken to be $Q_{x}=4.82$ and $Q_{y}=4.83$. The maximum COD occurs in the B6 quadrupole; the other smaller maxima occur in the B2 and C2 quadrupoles. Note that the COD outside the bump region is relatively small. 


\section{Vertical Bumps for the B6 Dump}

The vertical correction dipoles in Booster can be used to produce local vertical bumps at the B6 dump. These magnets have an integrated strength of $k=0.975 \times 10^{-4} \mathrm{Tm} / \mathrm{A}$ as reported by Thern [3], and can be excited with \pm 25 A maximum current. They produce a vertical kick

$$
\phi=k I /(B \rho)
$$

where $I$ is the excitation current. Table 2 lists the kicks at the maximum current $(25 \mathrm{~A})$ for protons with various kinetic energies. Figure 3 shows a

Table 2: Correction Dipole Kicks at $25 \mathrm{~A}$

\begin{tabular}{|c|c|c|}
\hline$W(\mathrm{MeV})$ & $B \rho(\mathrm{Tm})$ & $\phi(\mathrm{mrad})$ \\
\hline 200 & 2.149636 & 1.134 \\
400 & 3.183078 & 0.766 \\
600 & 4.066099 & 0.599 \\
800 & 4.881030 & 0.499 \\
1000 & 5.657373 & 0.431 \\
1200 & 6.409165 & 0.380 \\
1400 & 7.144162 & 0.341 \\
1600 & 7.867073 & 0.310 \\
1800 & 8.580951 & 0.284 \\
2000 & 9.287881 & 0.262 \\
\hline
\end{tabular}

vertical bump in red with the horizontal B6 dump bump in black. Here, again, the closed orbit was determined by the MAD code with the horizontal and vertical tunes taken to be $Q_{x}=4.82$ and $Q_{y}=4.83$. The four vertical correction dipoles are excited in the configuration, $-\mathrm{B} 1,+\mathrm{B} 3$, $+\mathrm{C} 3,-\mathrm{C} 5$, where the "+" and "-" signs denote upward and downward kicks respectively. This is the superposition of two Lambda Bumps which produces a maximum vertical COD in the B7 quadrupole. The other maxima occur in the B3 and C3 quadrupoles. The magnitude of the kicks is 1 milliradian.

Figure 4 shows a standard vertical three-bump (in red) centered on the B7 quadrupole. Here correction dipoles B5, B7, and C1 are excited to produce $+1.353,-0.827$, and +1.355 milliradian kicks respectively. 


\section{Booster Apertures and Acceptance Envelopes}

Let $X(s)$ and $Y(s)$ be the Horizontal and Vertical Half-Apertures at longitudinal position $s$ along the design orbit in Booster, and let $\beta_{x}(s)$ and $\beta_{y}(s)$ be the beta functions there. We then define

$$
E_{x}(s)=\frac{X^{2}}{\beta_{x}}, \quad E_{y}(s)=\frac{Y^{2}}{\beta_{y}}
$$

and denote by $e_{x}$ and $e_{y}$ the minimum values attained by $E_{x}(s)$ and $E_{y}(s)$ along the design orbit. These are the Horizontal and Vertical Acceptances of the machine. The values of $X$ and $Y$ at the positions where $E_{x}$ and $E_{y}$ reach their minimum values are the Limiting Apertures of the machine. The Horizontal and Vertical Acceptance Envelopes at position $s$ are given by

$$
H(s)= \pm \sqrt{e_{x} \beta_{x}(s)}, \quad V(s)= \pm \sqrt{e_{y} \beta_{y}(s)} .
$$

The horizontal and vertical apertures around the machine are given in Ref. [4]. Tables 3 and 4 list $X, \beta_{x}, E_{x}$ and $Y, \beta_{y}, E_{y}$ at various locations. (The values of $\beta_{x}$ and $\beta_{y}$ were determined by the MAD code with the horizontal and vertical tunes taken to be 4.82 and 4.83 ). Here we see that the horizontal and vertical acceptances are

$$
e_{x}=185, \quad e_{y}=90
$$

mm milliradians. The HLA (Horizontal Limiting Aperture) occurs at the septum of the F6 ejection magnet. The VLA (Vertical Limiting Aperture) occurs in dipole bending magnets 1,5 , and 7 of each superperiod. Close contenders for the HLA and VLA are respectively the septum of the C3 electrostatic inflector and the C3 kicker magnet. (Note that at the injection foil we assume the beam is centered in the available aperture.)

Table 3: Booster Horizontal Half-Apertures

\begin{tabular}{|c|c|c|c|c|}
\hline Location & In/Out & $X(\mathrm{~mm})$ & $\beta_{x}(\mathrm{~m})$ & $E_{x}$ \\
\hline Injection Foil & Inside & 50.0 & 10.77 & 232 \\
C3 Septum & Outside & 47.5 & 10.95 & 206 \\
F6 Septum & Outside & 47.0 & 11.97 & 185 \\
\hline
\end{tabular}


Table 4: Booster Vertical Half-Apertures

\begin{tabular}{|c|c|c|c|}
\hline Location & $Y(\mathrm{~mm})$ & $\beta_{y}(\mathrm{~m})$ & $E_{y}$ \\
\hline Dipole Bend 1 & 33 & 12.10 & 90.0 \\
Dipole Bend 5 & 33 & 12.12 & 89.8 \\
Dipole Bend 7 & 33 & 12.03 & 90.5 \\
C1 Kicker & 35 & 10.62 & 115 \\
C3 Kicker & 35 & 11.98 & 102 \\
C7 Kicker & 35 & 10.57 & 116 \\
D1 Kicker & 35 & 10.62 & 115 \\
\hline
\end{tabular}

\section{B6 Dump Position and Bump Requirements}

The design, dimensions, and placement of the B6 dump are the same as those of the D6 dump. As per John Hock and according to Drawing D36-M-1979, the length of the dump is 43.8125 inches (1.1128 meters) and the upstream end of the aperture is placed 29.0875 inches $(0.7388$ meters) downstream of the downstream face of the number 6 quadrupole.

\subsection{Horizontal Position}

The D6 dump is positioned horizontally (radially) so that the inside horizontal half-apertures at the upstream and downstream ends are

$$
A_{x}(u)=-42.1, \quad A_{x}(d)=-34.9
$$

$\mathrm{mm}$ with respect to the beam center line. The outside half-aperture is 50 mm at both ends. These numbers again come from John Hock and Drawing D36-M-1979. The inside apertures were presumably determined from calculations of the acceptance envelope in the machine, but this information apparently has been lost.

Ideally we would like to position the B6 dump so that the inside horizontal half-apertures at the upstream and downstream ends are

$$
A_{x}(u)=-\sqrt{e_{x} \beta_{x}(u)}, \quad A_{x}(d)=-\sqrt{e_{x} \beta_{x}(d)}-a_{x}
$$

where $e_{x}$ is the horizontal acceptance given in the previous section, $\beta_{x}(u)$ and $\beta_{x}(d)$ are the horizontal beta functions at the upstream and 
downstream ends, and $a_{x}$ is an offset added to ensure that beam hits the upstream end first. In this position, the inside aperture at the upstream end sits at the edge of the horizontal acceptance envelope (as determined by the limiting aperture at the F6 septum). Any horizontal COD to the inside at this point will make the dump the new HLA (assuming, of course, that the beam is centered in the available aperture everywhere else).

Taking the horizontal and vertical tunes to be $Q_{x}=4.82$ and $Q_{y}=4.83$, the MAD code gives $\beta_{x}(u)=10.43$ and $\beta_{x}(d)=7.19$ meters. With $e_{x}=185$ (mm milliradians) we then have

$$
A_{x}(u)=-43.9(\mathrm{~mm}), \quad A_{x}(d)+a_{x}=-36.5(\mathrm{~mm}) .
$$

These are respectively 1.8 and $1.6 \mathrm{~mm}$ further to the inside (away from the beam center line) than the original design values for the D6 dump. Initially, the B6 Dump will be positioned according to the original design. Later we may move toward the values given by (11) and (12) with an appropriate offset $a_{x}$ added to ensure that the beam hits the upstream end first.

\subsection{Horizontal Bump Requirements}

With the dump positioned horizontally according to equations (11) and (12), the minimum requirement for the horizontal dump bump is that it produce a small COD to the inside at the upstream end of the dump, thereby making the dump the HLA of the machine. The maximum requirement comes from consideration of the beam at extraction. As reported by Bleser [5], the horizontal acceptance of the $\mathrm{F} 6$ extraction channel is $30 \mathrm{~mm}$ milliradians (un-normalized). If the horizontal beam emittance at extraction is smaller than this, then in principle one can extract the beam without loss. If the emittance is larger, the excess beam must be scraped off elsewhere in the ring in order to avoid loss going through the extraction channel. Let us calculate the COD required to scrape off the excess beam at the upstream end of the B6 dump. Here the horizontal half-width of beam with emittance $\epsilon_{x}=30$ ( $\mathrm{mm}$ milliradians) is

$$
W_{x}(u)=\sqrt{\epsilon_{x} \beta_{x}(u)}
$$

and the required COD would be

$$
X=W_{x}(u)-A_{x}(u)=\left(\sqrt{\epsilon_{x}}-\sqrt{e_{x}}\right) \sqrt{\beta_{x}(u)}
$$


where $e_{x}=185$ (mm milliradians). With tunes $Q_{x}=4.82$ and $Q_{y}=4.83$ the MAD code gives $\beta_{x}(u)=10.43$ meters. Putting in these numbers we get $X=-26.2 \mathrm{~mm}$. The MAD code then finds that the string of 4 flat trims which produce the bump must be excited such that $\Delta B / B=0.01135$ in dipole $\mathrm{B} 2$. For protons with $2000 \mathrm{MeV}$ kinetic energy, the required current is $250 \mathrm{~A}$. Figures 5 and 6 show the resulting bump and beam envelopes. Here the black curve is the bump, the red curve is the horizontal beam envelope (with $\epsilon_{x}=30 \mathrm{~mm}$ milliradians), and the dashed blue curve is the horizontal acceptance envelope (as determined by the limiting aperture at the F6 septum). The inside and outside apertures of the dump according to the original design are outlined in black.

Figure 7 is a further enlargement of the dump region again showing the inside aperture according to the original design. Note that the aperture is actually quite close to the acceptance envelope we have calculated. The inside aperture according to equations (11) and (12) is shown in violet in Figure 8 with the upstream end just touching the acceptance envelope. The offset at the downstream end is taken to be $a_{x}=2 \mathrm{~mm}$ for illustration.

The ability to scrape off all of the beam on the dump is useful during fault and other studies. The required COD at the upstream end in this case is $X=-\sqrt{e_{x} \beta_{x}(u)}=-43.9 \mathrm{~mm}$. The MAD code then finds that $\Delta B / B=0.0191$ would be required in dipole $\mathrm{B} 2$ and the current for protons with $2000 \mathrm{MeV}$ kinetic energy would be $421 \mathrm{~A}$. Table 5 lists the COD at the upstream end of the dump for various values of $\Delta B / B$. The currents required for protons with $2000 \mathrm{MeV}$ kinetic energy are listed in the third column. Here we see that with the current limited to $300 \mathrm{~A}$ (steady state), the maximum COD is about $-31 \mathrm{~mm}$ at this energy. Scraping off all of the beam on the dump at this energy therefore requires a radial shift to the inside in addition to the dump bump.

\subsection{Vertical Position and Vertical Bump}

The D6 dump is positioned vertically so that the upper and lower half-apertures are +35 and $-35 \mathrm{~mm}$ with respect to the beam center line. These apertures are outlined in black in Figure 9. Here the dashed green curve is the vertical acceptance envelope as determined by the vertical limiting aperture in the number 1, 5, and 7 dipoles. The upper and lower apertures of the $\mathrm{B} 7$ dipole are outlined in violet.

Ideally we would like to position the B6 dump so that the upper aperture at the upstream and downstream ends sits at the edge of the vertical 
Table 5: Horizontal COD $(X)$ at the upstream end of the dump.

\begin{tabular}{|c|c|c|}
\hline$\Delta B / B$ & $X(\mathrm{~mm})$ & $\Delta I(\mathrm{~A})$ \\
\hline 0.004 & -9.24 & 88 \\
0.006 & -13.86 & 132 \\
0.008 & -18.46 & 176 \\
0.010 & -23.07 & 221 \\
0.012 & -27.66 & 265 \\
0.0136 & -31.33 & 300 \\
0.014 & -32.25 & 309 \\
0.016 & -36.84 & 353 \\
0.018 & -41.41 & 397 \\
0.020 & -45.99 & 441 \\
\hline
\end{tabular}

acceptance envelope. One might then hope to produce a local vertical bump that would make the dump the new VLA of the machine. However, if one uses the vertical correction dipoles to produce the bump, the resulting COD causes the beam to hit the upstream end of the B7 dipole first. This is illustrated in Figures 9 and 10. Here the black curve is the bump produced by correction dipoles B1, B3, C3, C5 as described in Section 4. The proton kinetic energy is taken to be $200 \mathrm{MeV}$ and the bump dipoles are excited with the maximum current of $25 \mathrm{~A}$. The red curve is the envelope of beam with a vertical emittance $\epsilon_{y}=23(\mathrm{~mm}$ milliradians). The upper and lower apertures of the B7 dipole are outlined in violet. In Figure 10 the upper aperture of the dump, outlined in violet, has been extended down to the vertical acceptance envelope. Here we see that the beam envelope hits the upstream end of the B7 dipole before it hits the upstream end of the dump.

In order to make the dump the new VLA of the machine, the aperture at the upstream end must be moved inside the original vertical acceptance envelope. This situation is depicted in Figure 11. Here the upper aperture, outlined in violet, has been moved $1 \mathrm{~mm}$ inside the original vertical acceptance envelope at the upstream end of the dump. The cost of this move is that the vertical acceptance is reduced from $e_{y}=90$ to $e_{y}=81$ (mm milliradians). One might hope to recover the lost acceptance with a vertical bump that moves the closed orbit downward away from the upper aperture. However, with such a bump, the resulting COD again causes the beam to hit the upstream end of the B7 dipole, this time on the 
lower aperture. This is illustrated in Figure 12. Here the dashed green curve is the original vertical acceptance envelope and the red curve is the envelope of beam that fills the original $e_{y}=90$ ( $\mathrm{mm}$ milliradians) acceptance. The black curve is a small vertical bump (again produced by correction dipoles B1, B3, C3, C5) which has been adjusted so that the beam just clears the upper aperture of the dump. The beam envelope then hits the lower aperture of the B7 dipole as indicated in the figure.

\section{References}

[1] K.A. Brown, et al., "Design of a Resonant Extraction System for the AGS Booster", Proceedings of the 1999 Particle Accelerator Conference, New York, 1999, pp. 1270-1272.

[2] R. Thern, "Booster Dipole Production Measurements", Booster Tech. Note No. 190, March 13, 1991.

[3] R. Thern, "Booster Ring Correction Magnets", Booster Tech. Note No. 224, May 20, 1994.

[4] A. Luccio, "Booster Chamber Aperture", Booster Tech. Note No. 202, November 13, 1991

[5] E. Bleser, unpublished notes. 


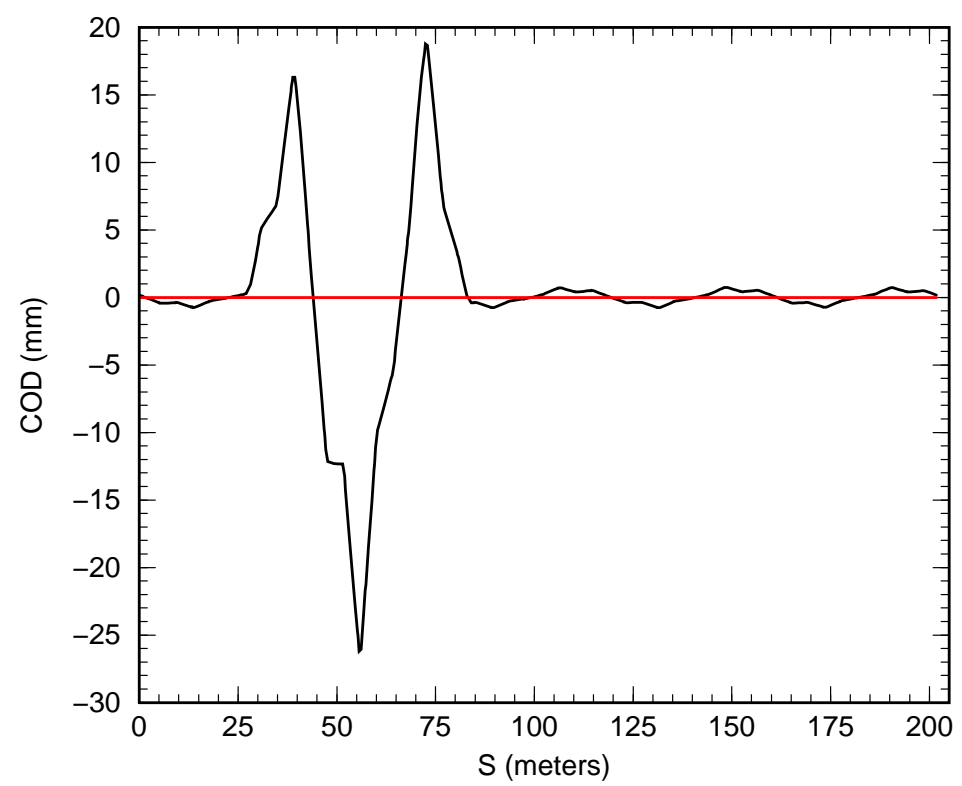

Figure 1: B6 Dump Bump: $\Delta B / B=0.01, Q_{x}=4.82, Q_{y}=4.83$

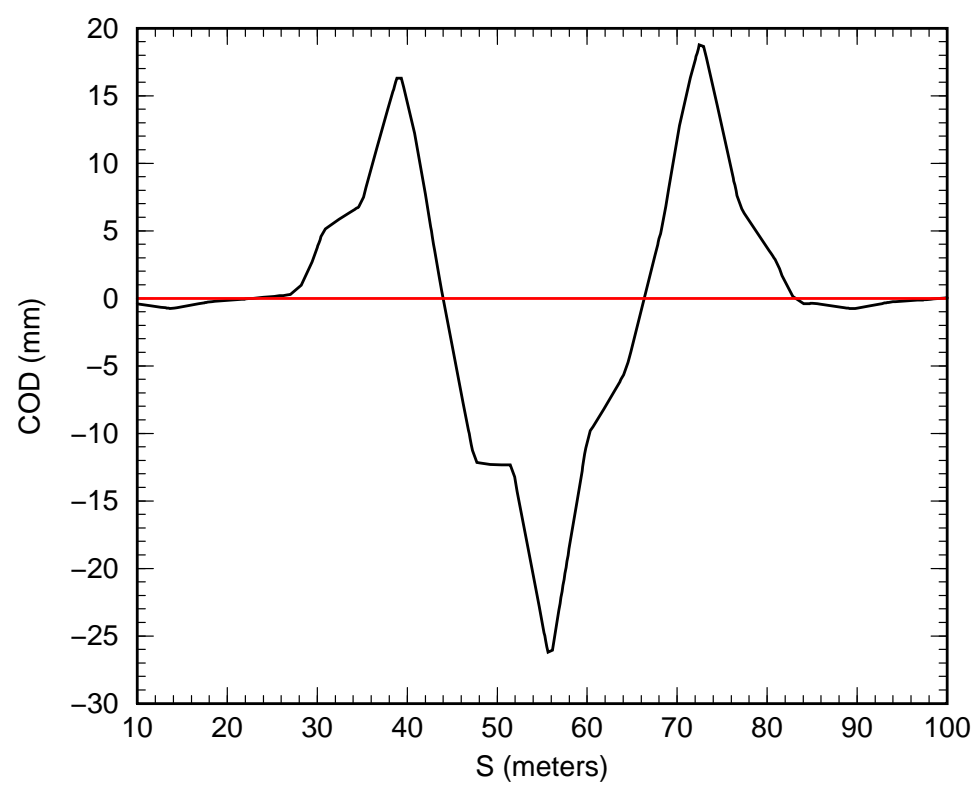

Figure 2: Enlarged view of bump region. 


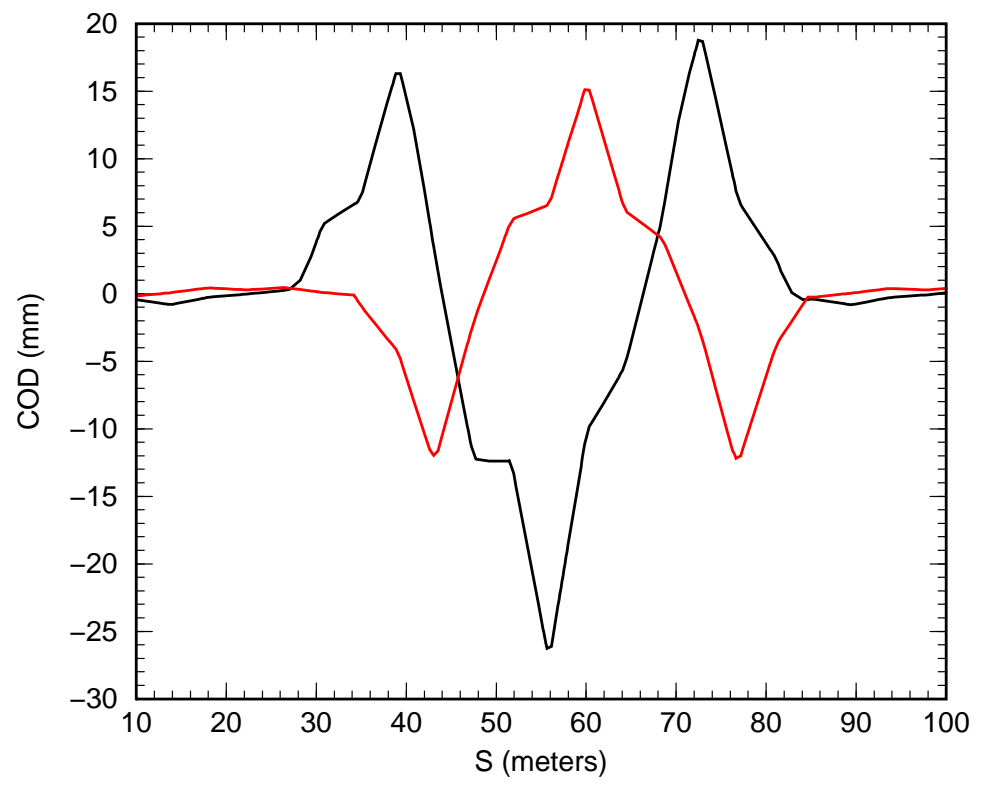

Figure 3: Red curve is superposition of two vertical lambda bumps (-B1, $+\mathrm{B} 3,+\mathrm{C} 3,-\mathrm{C} 5)$.

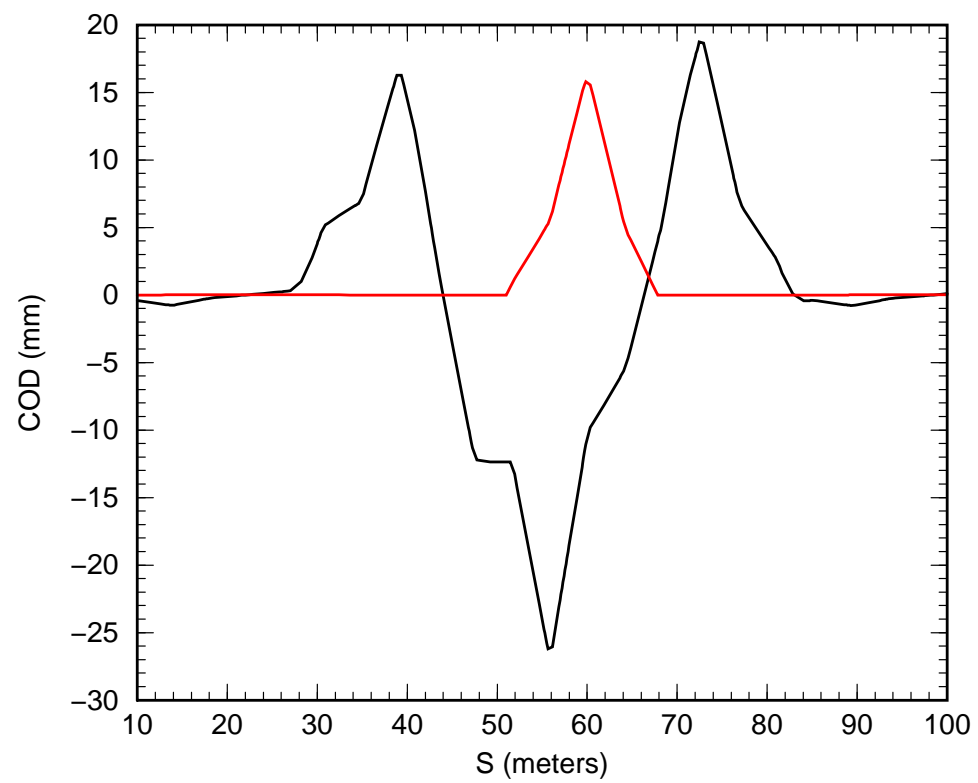

Figure 4: Red curve is vertical three-bump centered on quad B7. 


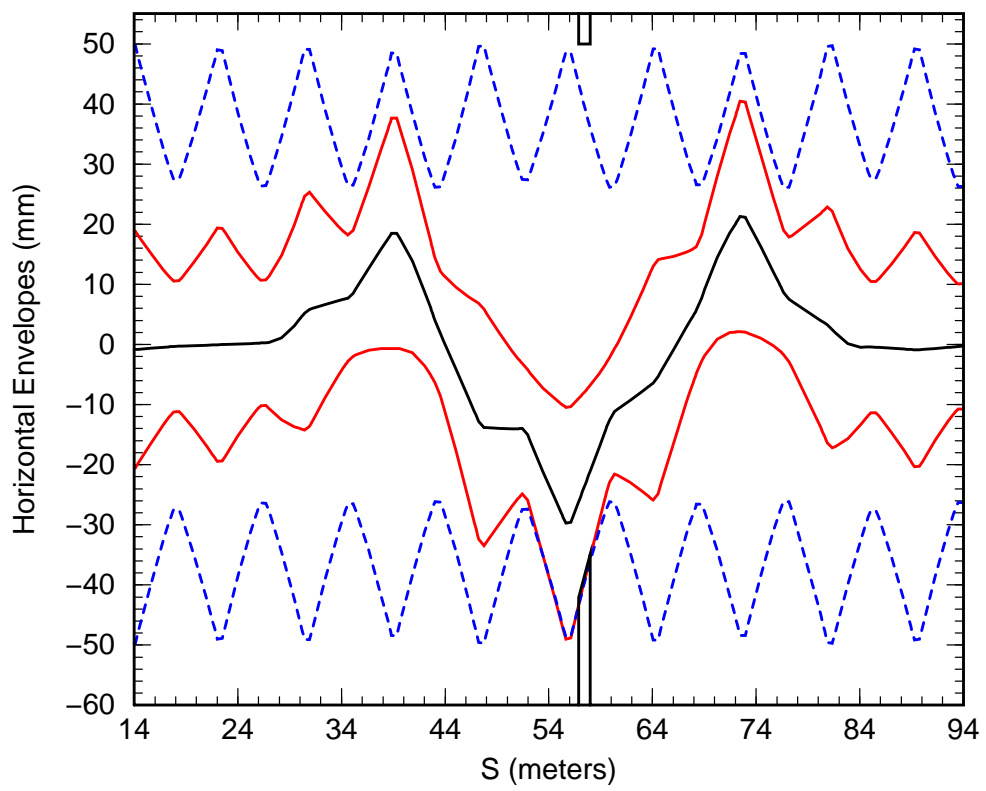

Figure 5: Horizontal dump bump, envelopes, and dump apertures.

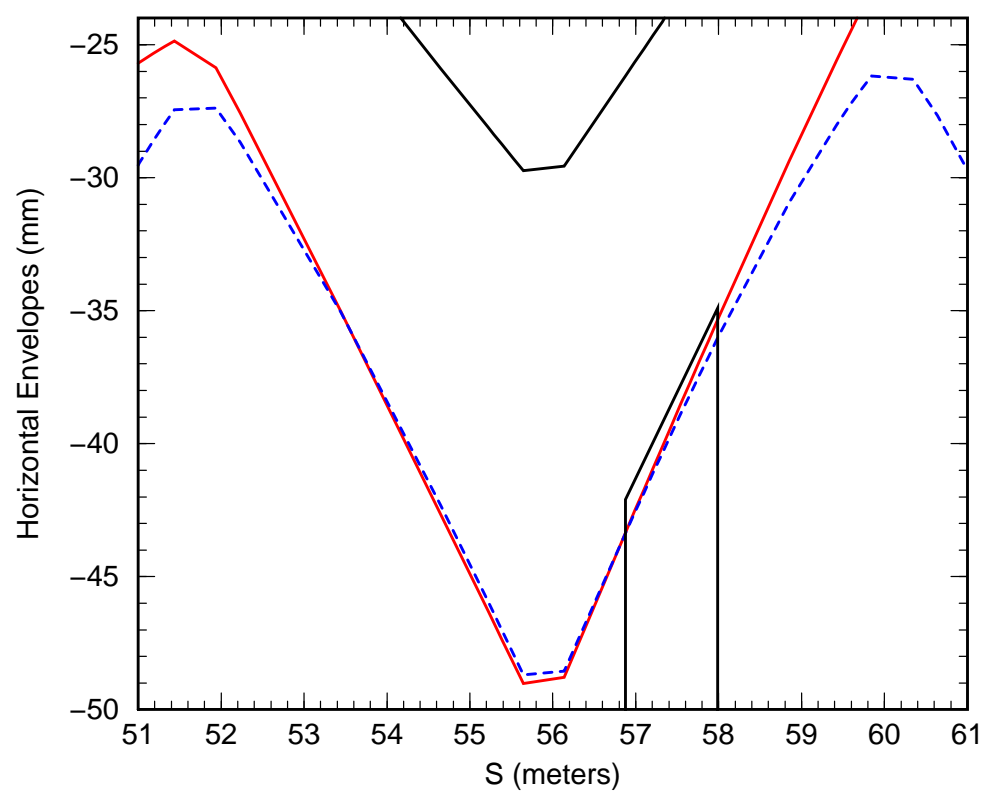

Figure 6: Enlarged view of dump region. Original dump aperture. 


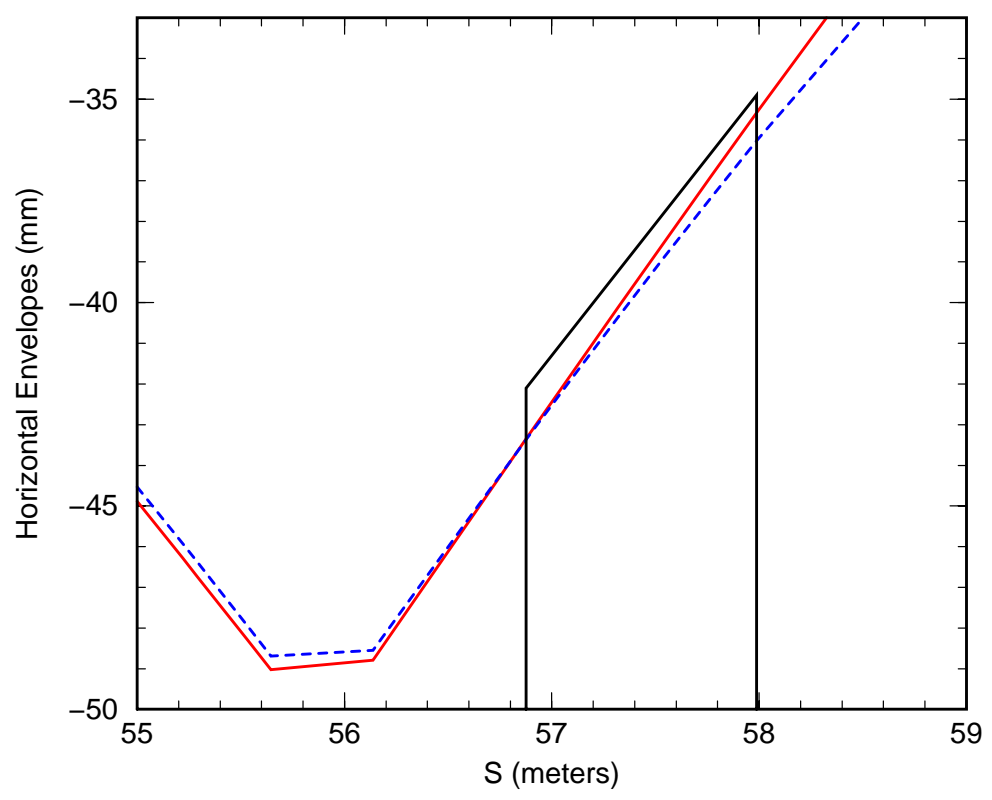

Figure 7: Further enlargement of dump region. Original dump aperture.

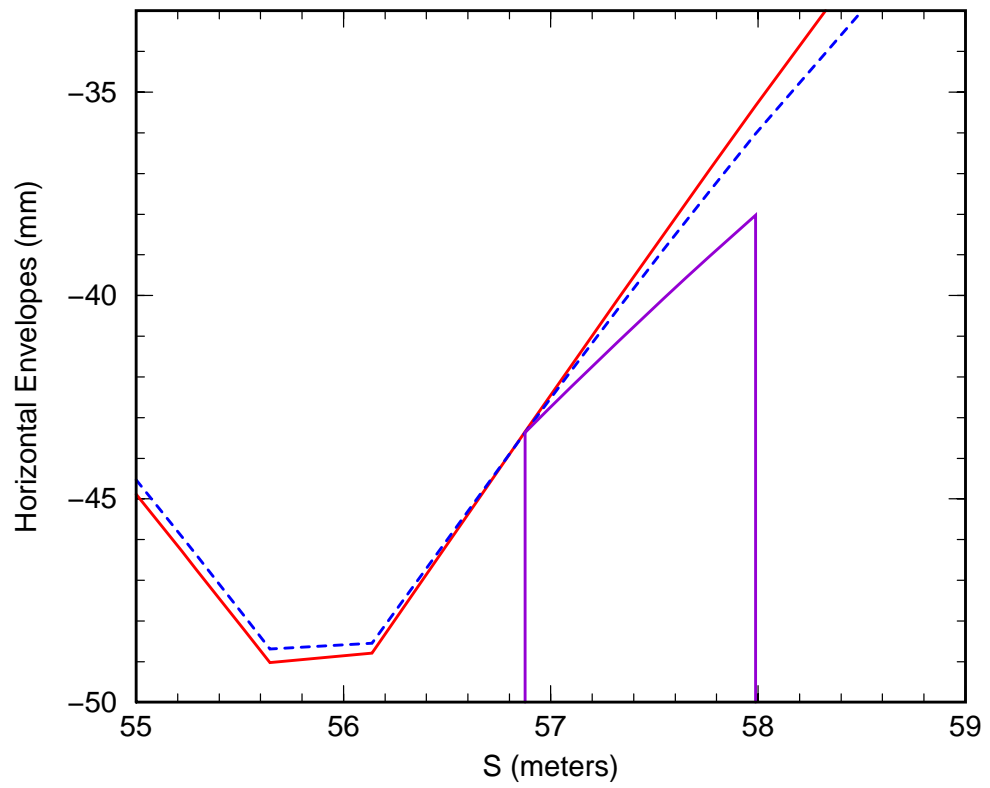

Figure 8: Dump aperture according to equations (11) and (12). 


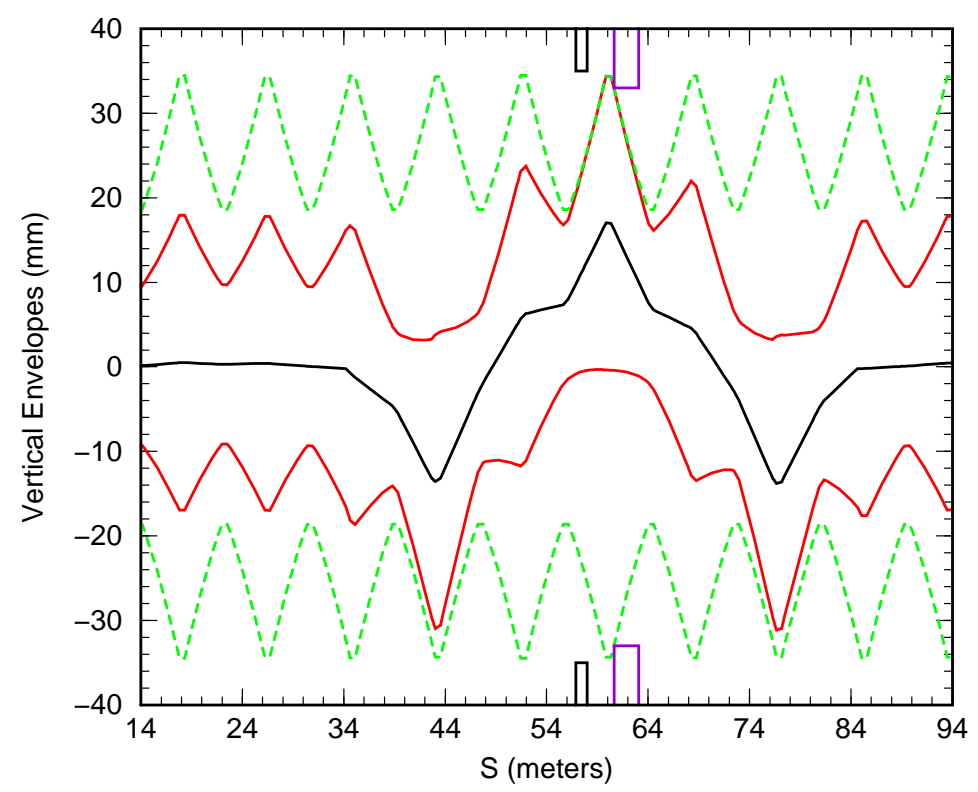

Figure 9: Vertical dump bump and envelopes; dump and dipole apertures.

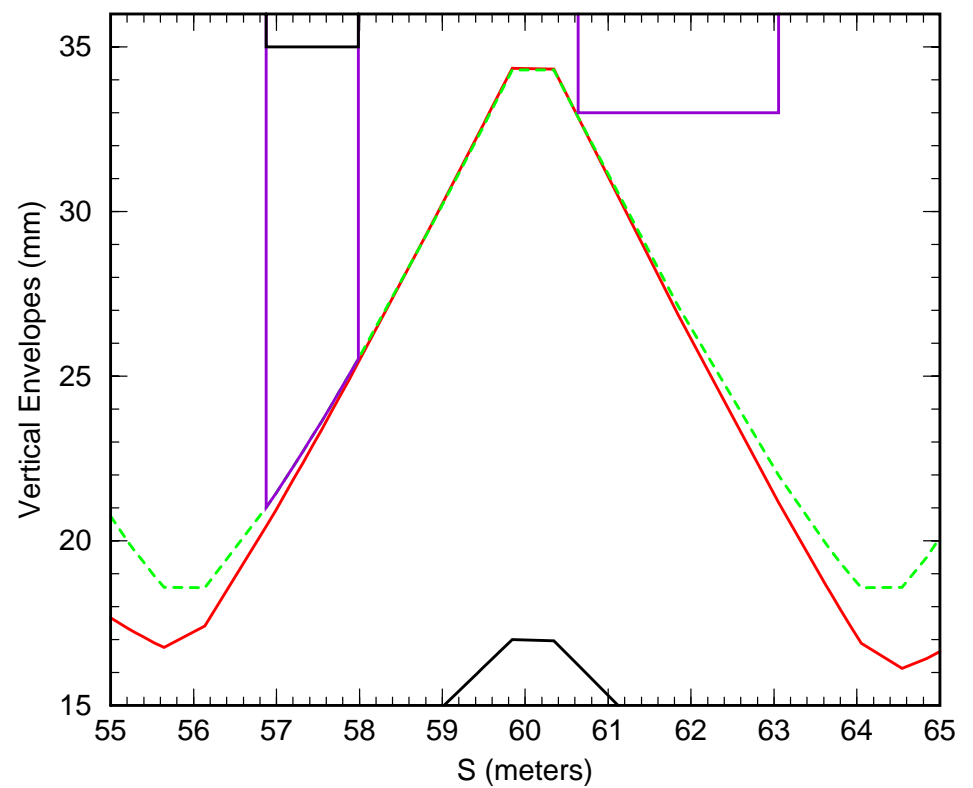

Figure 10: Enlarged view of dump region with extended aperture. 


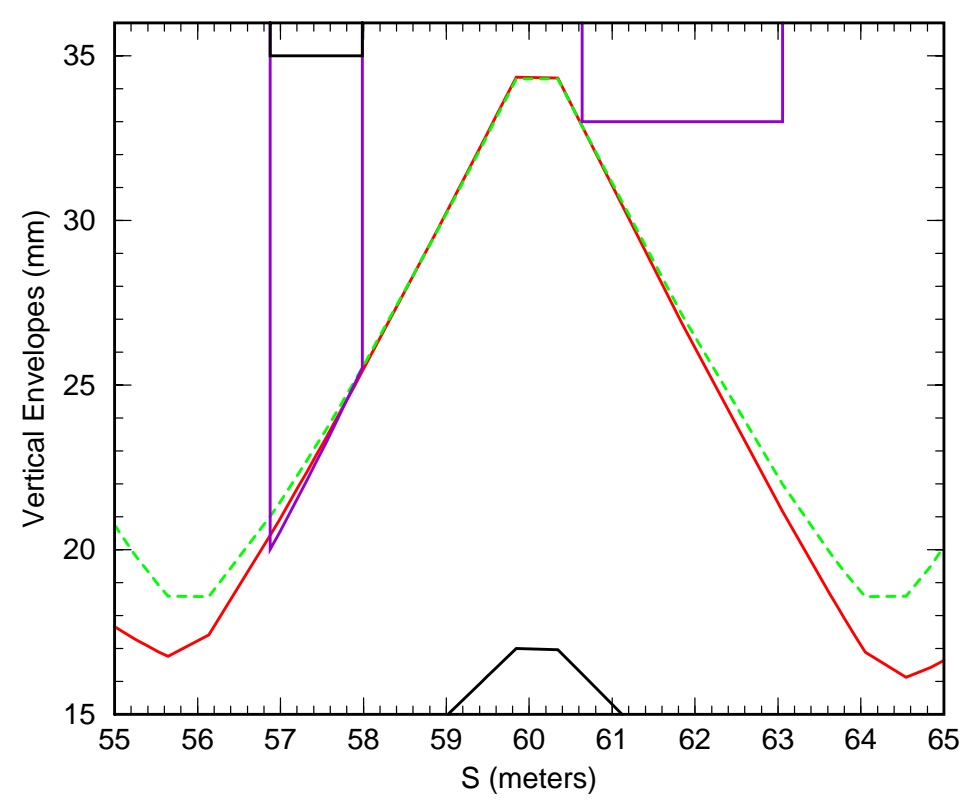

Figure 11: Aperture extended $1 \mathrm{~mm}$ inside the acceptance envelope.

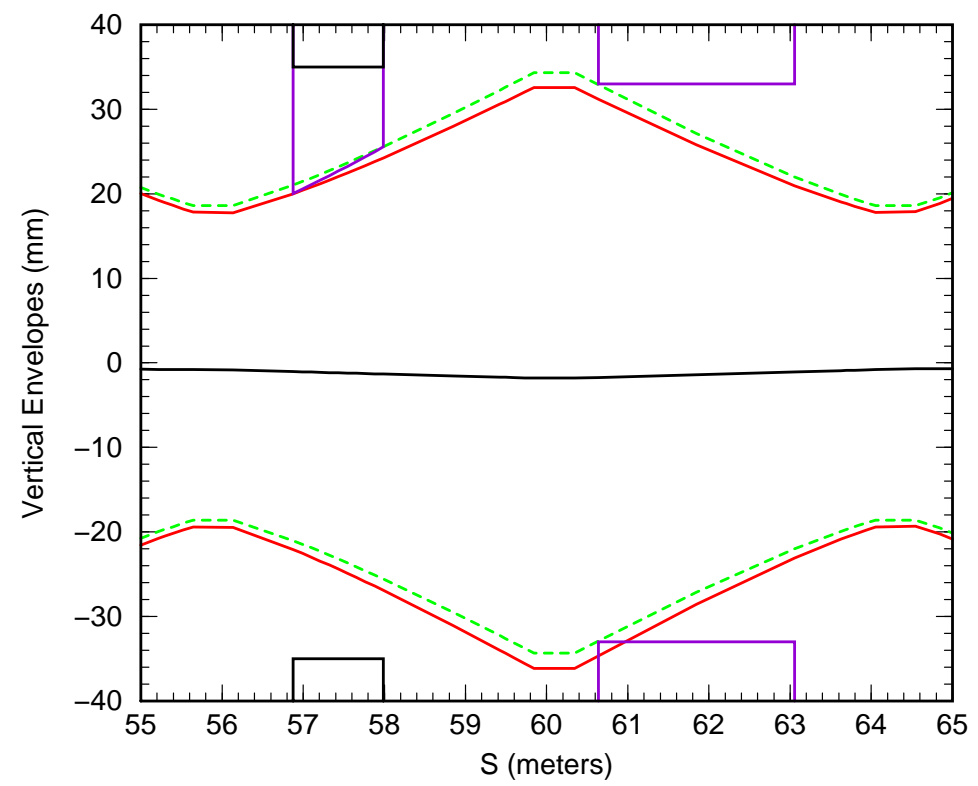

Figure 12: With a small downward bump, beam clears the dump aperture but hits dipole B7. 